\title{
On super-weakly compact sets and uniformly convexifiable sets
}

\author{
by \\ Lixin Cheng, Qinguin Cheng, Bo Wang and Wen Zhang (Xiamen)
}

\begin{abstract}
This paper mainly concerns the topological nature of uniformly convexifiable sets in general Banach spaces: A sufficient and necessary condition for a bounded closed convex set $C$ of a Banach space $X$ to be uniformly convexifiable (i.e. there exists an equivalent norm on $X$ which is uniformly convex on $C$ ) is that the set $C$ is super-weakly compact, which is defined using a generalization of finite representability. The proofs use appropriate versions of classical theorems, such as James' finite tree theorem, Enflo's renorming technique, Grothendieck's lemma and the Davis-Figiel-Johnson-Pełczyński lemma.
\end{abstract}

1. Introduction. This paper has several goals: (1) establishing a localized setting of the notion of super-reflexive Banach spaces, say, super-weakly compact sets; (2) searching for geometric and renorming features of superweakly compact sets; and (3) finding the topological nature of uniformly convexifiable sets.

Uniformly convexifiable sets. In many cases, the assumption of uniform convexity on the whole space is not natural. For example, if $T$ is a non-expansive mapping from a nonempty closed bounded convex set $C$ of a Banach space to itself, the Brouwer fixed point theorem (see, for instance, [11, [18]) says that the assumptions of weak compactness and of normal structure on $C$ guarantee that $T$ has a fixed point. This tells us that we only need to assume that $C$ has some kind of uniform convexity, that is, we only need a localized setting. A real-valued convex function $f$ defined on a convex set $C$ is said to be uniformly convex provided for every $\varepsilon>0$ there is $\delta>0$ such that $f(x)+f(y)-2 f((x+y) / 2) \geq \delta$ whenever $x, y \in C$ with $\|x-y\| \geq \varepsilon[3]$. The set $C$ is called uniformly convex (with respect to the norm $\|\cdot\|)$ provided for every $x_{0} \in C$ the function $f:=\left\|\cdot-x_{0}\right\|^{2}$ is uniformly convex on $C$. We say that $C$ is uniformly convexifiable if there is

2010 Mathematics Subject Classification: Primary 46B20, 46B03, 46B50.

Key words and phrases: finite representability, weakly compact set, uniformly convexifiable set, Banach space. 
an equivalent norm $|\cdot|$ on $X$ such that $C$ is uniformly convex with respect to $|\cdot|$.

QUESTION A. What is the topological nature of closed bounded convex sets which are uniformly convexifiable?

Super-weakly compact sets. Super-reflexive or uniformly convexifiable Banach spaces play an important role in both Banach space theory and its applications (see, for instance, [1-2], [4], 66-8], [10], [13], [15]17], and [19]), and they form an extremely useful class of reflexive spaces. The deep Enflo-Pisier renorming theorem (see [9] and [20]) states that every superreflexive Banach space is uniformly convexifiable and vice versa. Also, much progress has been made in the theory of weakly compact sets. This notion can be viewed as a generalization and localization of reflexive spaces since a Banach space is reflexive if and only if its closed unit ball is weakly compact. For theoretical symmetry or completion, we need a reasonable notion of "super-weakly compact sets" which would guarantee the following conclusion: A Banach space is super-reflexive if and only if its closed unit ball is super-weakly compact. We define "super-weak compactness" as follows: A nonempty weakly closed convex set $C$ is said to be super-weakly compact if every set $D$ which is finitely representable in $C$ is relatively weakly compact.

QUeSTION B. Is every super-weakly compact convex set uniformly convexifiable?

This article focuses on the two questions above. As a result, we show the following main theorem.

Main TheORem. A closed convex bounded set is uniformly convexifiable if and only if it is super-weakly compact.

This paper is divided into four sections and organized as follows. In Section 2, we first generalize the notion of finite representability between two Banach spaces to that between two convex subsets, and this is done by substituting simplexes for finite-dimensional subspaces; second, we introduce the notion of super-weakly compact sets in terms of the generalized finite representability; and finally, we show that an analogue of James' characterization for super-reflexive spaces holds for super-weakly compact sets. We state it as follows.

Theorem 2.14. Let $C$ be a bounded closed convex set in a Banach space $(X,\|\cdot\|)$. Then the following assertions are equivalent:

(i) $C$ is not super-weakly compact.

(ii) $C$ has the finite tree property. 
(iii) There exists $\theta>0$ such that for every $n \in \mathbb{N}$ there are $x_{1}, \ldots, x_{n} \in C$ such that for all $1 \leq k \leq n-1$,

$$
\operatorname{dist}\left(\operatorname{co}\left\{x_{1}, \ldots, x_{k}\right\}, \operatorname{co}\left\{x_{k+1}, \ldots, x_{n}\right\}\right)>\theta \text {. }
$$

In Section 3, by generalizing a series of Enflo's lemmas [9] to general super-weakly compact convex sets, we show that a bounded closed convex set is super-weakly compact if and only if for every $\varepsilon>0$, there exists a bounded $\varepsilon$-uniformly convex function on it; and further, the image of a super-weakly compact convex set under a uniformly continuous affine mapping is again super-weakly compact. These statements, in turn, imply that the product $A \times B$ and the difference $A-B$ of any two super-weakly compact convex sets $A$ and $B$ are again super-weakly compact.

In Section 4, we first verify that Grothendieck's lemma for weakly compact sets is still valid for super-weakly compact sets:

Lemma 4.5. A closed convex subset $K$ of a Banach space $X$ is superweakly compact (if and only) if for every $\varepsilon>0$ there exists a super-weakly compact convex set $K_{\varepsilon}$ such that $K \subset K_{\varepsilon}+\varepsilon B_{X}$, where $B_{X}$ denotes the closed unit ball of $X$.

Then employing this result, a refined version of the Davis-Figiel-JohnsonPełczyński Lemma [5] and theorems in the previous sections, we finally show the main theorem.

In this paper, the letter $X$ will always denote a real Banach space and $X^{*}$ its dual. We denote by $B_{X}$ the closed unit ball of $X$, and by $S_{X}$ the unit sphere. For a set $A$ in $X, \operatorname{co} A, \operatorname{aff} A$ and $\operatorname{span} A$ stand for the convex hull, affine hull and linear hull of the set $A$, respectively, and $\overline{\operatorname{co}} A$ and $\overline{\operatorname{aff}} A$ are the respective closures.

2. Properties of super-weakly compact sets. In this section, we first introduce a notion of finite representability between two convex sets of Banach spaces, making use of simplexes and affine mappings. Then in terms of the generalized finite representability we introduce the notion of superweakly compact sets, and discuss their finite tree property (Theorem 2.14). We will see that a super-weakly compact convex set behaves just like the closed unit ball of a super-reflexive space. Therefore, this concept can be viewed as a generalization and localization of the notion of super-reflexivity of Banach spaces.

To start with, we recall the classical notion of finite representability introduced by James (see [13] and [14]), which has played an important role in studying various "super-properties" of Banach spaces.

Definition 2.1. Suppose that $X$ and $Y$ are Banach spaces. We say that $X$ is finitely representable in $Y$ if for every $\varepsilon>0$ and every finite-dimensional 
subspace $M \subset X$, there exist a subspace $N \subset Y$ and a linear mapping $T: M \rightarrow N$ such that $\|T\| \cdot\left\|T^{-1}\right\| \leq 1+\varepsilon$.

For generalizing this notion to convex sets, a natural way is to substitute simplexes and affine mappings for linear subspaces and linear mappings.

Definition 2.2. Suppose that $\left\{x_{i}\right\}_{i=0}^{n}$ are $n+1$ vectors in $X$.

(i) $\left\{x_{i}\right\}_{i=0}^{n}$ are said to be affinely independent if $\left\{x_{i}-x_{0}\right\}_{i=1}^{n}$ are linearly independent.

(ii) $\operatorname{co}\left\{x_{i}\right\}_{i=0}^{n}$ is called an $n$-simplex in $X$ with vertices at $x_{i}$ if $\left\{x_{i}\right\}_{i=0}^{n}$ are affinely independent.

Definition 2.3. Suppose $A \subset X$ and $B \subset Y$ are convex subsets.

(i) A mapping $T: A \rightarrow B$ is called affine if $T(\lambda x+(1-\lambda) y)=\lambda T(x)+(1-\lambda) T(y) \quad$ for all $x, y \in A$ and $\lambda \in[0,1]$.

(ii) An affine mapping $T: A \rightarrow B$ is called a $(1+\varepsilon)$-affine embedding from $A$ to $B$, for some $\varepsilon>0$, if $(1-\varepsilon)\|x-y\| \leq\|T(x)-T(y)\| \leq(1+\varepsilon)\|x-y\| \quad$ for all $x, y \in A$. If such a map $T$ exists, then we say that $A$ can be $(1+\varepsilon)$-affinely embedded into $B$.

In particular, if $A=X, B=Y$, then a linear map $T$ from $X$ into $Y$ is called a $(1+\varepsilon)$-linear embedding from $X$ to $Y$ if $T$ satisfies the inequalities above for all $x, y \in X$. In this case, $X$ is said to be $(1+\varepsilon)$-linearly embedded into $Y$.

Now we generalize the notion of finite representability as follows.

Definition 2.4. Suppose that $A \subset X$ and $B \subset Y$ are nonempty convex subsets. We say that $A$ is finitely representable in $B$ (for short: $A \stackrel{\text { fr }}{\hookrightarrow} B$ ) if for every $\varepsilon>0$, each $n$-simplex $S(A)$ in $A$ can be $(1+\varepsilon)$-affinely embedded into $B$.

Clearly, every convex subset of $A$ is finitely representable in $A$.

Proposition 2.5. Suppose that $A \subset X, B \subset Y$ and $C \subset Z$ are nonempty convex subsets. Then:

(i) $A \stackrel{\text { fr }}{\hookrightarrow} B$ and $B \stackrel{\text { fr }}{\hookrightarrow} C \Rightarrow A \stackrel{\text { fr }}{\hookrightarrow} C$.

(ii) For every $x_{0} \in A$ and $y_{0} \in B, A \stackrel{\mathrm{fr}}{\hookrightarrow} B \Leftrightarrow A-x_{0} \stackrel{\mathrm{fr}}{\hookrightarrow} B-y_{0}$.

(iii) $A \stackrel{\text { fr }}{\hookrightarrow} B \Leftrightarrow \bar{A} \stackrel{\text { fr }}{\hookrightarrow} B$.

Proof. (i) and (ii) easily follow from the definition of finite representability. To prove (iii), it suffices to show the necessity. By (i), we need only show $\bar{A} \stackrel{\text { f.r }}{\hookrightarrow} A$. For every $\varepsilon>0$ and every $n$-simplex $C_{n}=\operatorname{co}\left\{x_{0}, x_{1}, \ldots, x_{n}\right\} \subset \bar{A}$, 
let $E=\operatorname{span}\left\{x_{i}-x_{0}\right\}_{i=1}^{n}$. Let $\|\cdot\|$ be the norm on $X$. Since $(E,\|\cdot\|)$ is homeomorphic to $\left(l_{1}^{n},\|\cdot\|_{1}\right)$, we can find a positive number $\beta$ such that

$\beta \sum_{i=1}^{n}\left|\alpha_{i}\right|=\beta\left\|\left(\alpha_{1}, \ldots, \alpha_{n}\right)\right\|_{1} \leq\left\|\sum_{i=1}^{n} \alpha_{i}\left(x_{i}-x_{0}\right)\right\| \quad$ for all $\left(\alpha_{1}, \ldots, \alpha_{n}\right) \in l_{1}^{n}$.

The density of $A$ in $\bar{A}$ implies that for $\alpha=\frac{1}{2} \beta \varepsilon$, there is a sequence $\left\{x_{i}^{1}\right\}_{i=0}^{n} \subset$ $A$ with

$$
\left\|x_{i}^{1}-x_{i}\right\|<\alpha \quad \text { for } i=0,1, \ldots, n .
$$

Set $C_{n}^{1}=\operatorname{co}\left\{x_{0}^{1}, x_{1}^{1}, \ldots, x_{n}^{1}\right\}$. We claim that the mapping $T: C_{n} \rightarrow C_{n}^{1}$ defined by $T\left(x_{i}\right)=x_{i}^{1}$ for $i=0,1, \ldots, n$ is a $(1+\varepsilon)$-affine embedding. To see this, let $x, y$ be in $C_{n}$; then there exist unique $\alpha_{i}, \beta_{i} \geq 0$ with $\sum_{i=0}^{n} \alpha_{i}=1$ and $\sum_{i=0}^{n} \beta_{i}=1$ such that

$$
x=\sum_{i=0}^{n} \alpha_{i} x_{i}, \quad y=\sum_{i=0}^{n} \beta_{i} x_{i} .
$$

Thus

$$
\begin{aligned}
\mid \| T(x)-T(y) & \|-\| x-y \| \mid \\
& =\left|\left\|\sum_{i=1}^{n}\left(\alpha_{i}-\beta_{i}\right)\left(x_{i}^{1}-x_{0}^{1}\right)\right\|-\left\|\sum_{i=1}^{n}\left(\alpha_{i}-\beta_{i}\right)\left(x_{i}-x_{0}\right)\right\|\right| \\
& \leq \sum_{i=1}^{n}\left|\alpha_{i}-\beta_{i}\right|\left(\left\|x_{i}^{1}-x_{i}\right\|+\left\|x_{0}^{1}-x_{0}\right\|\right) \\
& \leq \frac{2 \alpha}{\beta}\left\|\sum_{i=1}^{n}\left(\alpha_{i}-\beta_{i}\right)\left(x_{i}-x_{0}\right)\right\|=\varepsilon\|x-y\| .
\end{aligned}
$$

This proves our claim.

Lemma 2.6. Suppose that $A \subset X$ and $B \subset Y$ are nonempty convex subsets, set $E=\operatorname{aff} A, F=$ aff $B$, and suppose that $A$ has nonempty relative interior in $E$ (denoted by $\operatorname{int}_{E} A$ ). If $A$ can be $\left(1+\varepsilon_{0}\right)$-affinely embedded into $B$ for some $\varepsilon_{0} \geq 0$, then $E$ can be $\left(1+\varepsilon_{0}\right)$-affinely embedded into $F$.

Proof. Choose any $a \in \operatorname{int}_{E} A$ and set $A_{1}=A-a$ and $E_{1}=$ aff $A_{1}$ $\left(=\operatorname{span} A_{1}\right)$. Then $0 \in \operatorname{int}_{E_{1}} A_{1}$. Let $T: E \rightarrow F$ be a $\left(1+\varepsilon_{0}\right)$-affine embedding from $A$ to $B$. Then $T_{1}: E_{1} \rightarrow F_{1}$ defined by

$$
T_{1}(x)=T(x+a)-T(a) \quad \text { for all } x \in E_{1}
$$

is a $\left(1+\varepsilon_{0}\right)$-linear embedding from $A_{1}$ to $B_{1}$, where $B_{1}=B-T a$ and $F_{1}=$ aff $B_{1}=\operatorname{span} B_{1}$. Therefore, $T_{1}$ is also a $\left(1+\varepsilon_{0}\right)$-linear embedding from $A_{1}$ to $F_{1}$. This implies that $T_{1}$ is again a $\left(1+\varepsilon_{0}\right)$-linear embedding from $E_{1}\left(=\bigcup_{n=1}^{\infty} n A_{1}\right)$ to $F_{1}$. Thus, $T$ is a $\left(1+\varepsilon_{0}\right)$-affine embedding from $E\left(=E_{1}+a\right)$ to $F\left(=F_{1}+T a\right)$. 
By Lemma 2.6 and a simple argument on linear homeomorphism, we get the following results.

Proposition 2.7. Suppose that $X$ and $Y$ are Banach spaces and assume that $A \subset X, B \subset Y$ are nonempty convex subsets. Then:

(i) $X \stackrel{\text { fr }}{\hookrightarrow} Y \Leftrightarrow B_{X} \stackrel{\text { fr }}{\hookrightarrow} B_{Y}$.

(ii) $A \stackrel{\text { fr }}{\hookrightarrow} B \Rightarrow$ aff $A \stackrel{\text { fr }}{\hookrightarrow}$ aff $B$.

The following counter-example shows that the converse of Proposition 2.7 (ii) is not true.

Example 2.8. Suppose that $X$ is a separable nonreflexive Banach space and $\left\{x_{n}\right\}$ is a dense sequence in the unit ball $B_{X}$. Let $A=\overline{\operatorname{co}}\left\{ \pm x_{n} / n\right\}$. Then $A$ is a compact convex set in $X$. Clearly, aff $A=\operatorname{span} A$ is a dense subspace of $X$, and $X$ is not finitely representable in $A$. But by Proposition 2.5(iii), $X \stackrel{\text { fr }}{\hookrightarrow}$ aff $A$.

Definition 2.9. A Banach space $X$ is said to be super-reflexive if every Banach space $Y$ whose unit ball is finitely representable in $B_{X}$ is reflexive.

Now we state the definition of super-weakly compact convex sets.

Definition 2.10. A bounded closed convex set $C$ of $X$ is said to be super-weakly compact if every convex set $D$ which is finitely representable in $C$ is relatively weakly compact.

To study super-weakly compact sets, we need two notions concerning $\varepsilon$-trees.

Definition 2.11. Suppose that $X$ is a Banach space, $\varepsilon>0$, and for $n \in \mathbb{N}, A_{n}, A \subset X$ are given by

$$
A_{n}=\left\{x_{\varepsilon_{1}, \ldots, \varepsilon_{n}}: \varepsilon_{i}=1,2 \text { and } i=1, \ldots, n\right\}, \quad A=\bigcup_{n=1}^{\infty} A_{n} .
$$

(i) The subset $A_{n}$ is called an $(n, \varepsilon)$-tree if

$$
x_{\varepsilon_{1}, \ldots, \varepsilon_{k}}=\frac{1}{2}\left(x_{\varepsilon_{1}, \ldots, \varepsilon_{k}, 1}+x_{\varepsilon_{1}, \ldots, \varepsilon_{k}, 2}\right)
$$

and

$$
\left\|x_{\varepsilon_{1}, \ldots, \varepsilon_{k}, 1}-x_{\varepsilon_{1}, \ldots, \varepsilon_{k}, 2}\right\| \geq \varepsilon
$$

for $k=1, \ldots, n-1$.

(ii) The subset $A$ is said to be an $(\infty, \varepsilon)$-tree if $A_{n}$ is an $(n, \varepsilon)$-tree for all $n \in \mathbb{N}$.

Definition 2.12. Suppose that $C$ is a nonempty convex subset of a Banach space $X$. Then we say that 
(i) $C$ has the finite tree property if there is $\varepsilon>0$ such that for every $n \in \mathbb{N}$ there exists an $(n, \varepsilon)$-tree in $C$;

(ii) $C$ has the infinite tree property if for some $\varepsilon>0$ there exists an $(\infty, \varepsilon)$-tree in $C$.

Definitions 2.11 and 2.12 were introduced by James in 1972 [14] in order to characterize super-reflexive spaces. The James characterization of superreflexive spaces says that a Banach space is super-reflexive if and only if its closed unit ball does not have the finite tree property. We will generalize this characterization to super-weakly compact convex subsets (Theorem 2.14). For this purpose, we need the following lemma which was motivated by [14].

Lemma 2.13. Suppose that $C \subset X$ is a nonempty convex set. If $C$ has the finite tree property, then there exist a Banach space $E$ and a convex set $A$ in $E$ such that $A$ has the infinite tree property and $\bar{A} \stackrel{\mathrm{fr}}{\hookrightarrow} C$.

Proof. Since $C$ has the finite tree property, there exists some $\varepsilon>0$ such that for every $n \in \mathbb{N}$ we can find, in $C$, an $(n, \varepsilon)$-tree

$$
G_{n}=\left\{x_{\varepsilon_{1}, \ldots, \varepsilon_{n}}^{(n)}: \varepsilon_{i}=1,2 \text { and } i=1, \ldots, n\right\} .
$$

Choose an infinite sequence of symbols

$$
S \equiv\left\{\xi_{\varepsilon_{1}, \ldots, \varepsilon_{n}}: n \in \mathbb{N}, \varepsilon_{i}=1,2 \text { and } i=1, \ldots, n\right\}
$$

and let $Y=\operatorname{span} S$. Let $\mathscr{U}$ be a nonprincipal ultrafilter on the positive integers $\mathbb{N}$. Then for every $r \in \mathbb{N}$ and every set of $2^{r}$ rational numbers $\left\{\lambda_{\varepsilon_{1}, \ldots, \varepsilon_{r}}: \varepsilon_{i}=1,2\right\}$, the following limit exists:

$$
\lim _{\mathscr{U}}\left\|\sum_{\varepsilon_{i}=1,2 ; 1 \leq i \leq r} \lambda_{\varepsilon_{1}, \ldots, \varepsilon_{i}} x_{\varepsilon_{1}, \ldots, \varepsilon_{i}}^{(n)}\right\| .
$$

Set

$$
D=\left\{\sum_{\varepsilon_{i}=1,2 ; 1 \leq i \leq r} \lambda_{\varepsilon_{1}, \ldots, \varepsilon_{i}} \xi_{\varepsilon_{1}, \ldots, \varepsilon_{i}}: \forall r \in \mathbb{N}, \lambda_{\varepsilon_{1}, \ldots, \varepsilon_{i}} \in \mathbb{Q}\right\}
$$

and let

$$
\left|\sum_{\varepsilon_{j}=1,2 ; 1 \leq j \leq r} \lambda_{\varepsilon_{1}, \ldots, \varepsilon_{j}} \xi_{\varepsilon_{1}, \ldots, \varepsilon_{j}}\right|=\lim _{\mathscr{U}}\left\|\sum_{\varepsilon_{j}=1,2 ; 1 \leq j \leq r} \lambda_{\varepsilon_{1}, \ldots, \varepsilon_{j}} x_{\varepsilon_{1}, \ldots, \varepsilon_{j}}^{(n)}\right\| .
$$

This defines a function $|\cdot|$ on $D$. We extend $|\cdot|$ to $Y$, keeping the notation $|\cdot|$. It is easy to see that $|\cdot|$ is a seminorm on $Y$. Let $N=\{x \in X:|x|=0\}$. Then the quotient space $F:=Y / N$ endowed with the quotient norm $\|\cdot\|_{F}$ corresponding to $|\cdot|$ is a normed space. We denote by $E$ the completion of $\left(F,\|\cdot\|_{F}\right)$, and by $\bar{\xi}$ the quotient vector of $\xi$, i.e., $\bar{\xi}=\xi+N$ for every $\xi$ in $Y$. We claim that

$$
B:=\left\{\bar{\xi}_{\varepsilon_{1}, \ldots, \varepsilon_{n}}: n \in \mathbb{N}, \varepsilon_{i}=1,2 \text { and } i=1, \ldots, n\right\}
$$


is an $(\infty, \varepsilon)$-tree. To see this, it suffices to note that for every $n \in \mathbb{N}, G_{n}$ is an $(n, \varepsilon)$-tree and (2.3) holds, thus for every $m \in \mathbb{N}, \varepsilon_{i}=1,2$ and $i=1, \ldots, m$,

$$
\begin{aligned}
\| \bar{\xi}_{\varepsilon_{1}, \ldots, \varepsilon_{m}}-\frac{1}{2}\left(\bar{\xi}_{\varepsilon_{1}, \ldots, \varepsilon_{m}, 1}+\bar{\xi}_{\varepsilon_{1}, \ldots, \varepsilon_{m}, 2}\right) & \|_{F} \\
=\lim _{\mathscr{U}}\left\|x_{\varepsilon_{1}, \ldots, \varepsilon_{m}}^{(n)}-\frac{1}{2}\left(x_{\varepsilon_{1}, \ldots, \varepsilon_{m}, 1}^{(n)}+x_{\varepsilon_{1}, \ldots, \varepsilon_{m}, 2}^{(n)}\right)\right\| & =0
\end{aligned}
$$

and

$$
\left\|\bar{\xi}_{\varepsilon_{1}, \ldots, \varepsilon_{m}, 1}-\bar{\xi}_{\varepsilon_{1}, \ldots, \varepsilon_{m}, 2}\right\|_{F}=\lim _{\mathscr{U}}\left\|x_{\varepsilon_{1}, \ldots, \varepsilon_{m}, 1}^{(n)}-x_{\varepsilon_{1}, \ldots, \varepsilon_{m}, 2}^{(n)}\right\| \geq \varepsilon
$$

(2.4) and (2.5) say that $B$ is an $(\infty, \varepsilon)$-tree, and hence the convex set $A:=$ co $B$ has the infinite tree property. Clearly, $A$ is finitely representable in $C$ by the definition of the seminorm $|\cdot|$ on $Y$. Proposition 2.5(iii) gives $\bar{A} \stackrel{\mathrm{fr}}{\hookrightarrow} C$.

Now, we prove our characterization of super-weakly compact sets.

Theorem 2.14. Let $C$ be a bounded closed convex set in a Banach space $(X,\|\cdot\|)$. Then the following assertions are equivalent:

(i) $C$ is not super-weakly compact.

(ii) $C$ has the finite tree property.

(iii) There exists $\theta>0$ such that for every $n \in \mathbb{N}$ there are $x_{1}, \ldots, x_{n} \in C$ such that for all $1 \leq k \leq n-1$,

$$
\operatorname{dist}\left(\operatorname{co}\left\{x_{1}, \ldots, x_{k}\right\}, \operatorname{co}\left\{x_{k+1}, \ldots, x_{n}\right\}\right)>\theta .
$$

Proof. (i) $\Rightarrow$ (iii). Assume that $C$ is not super-weakly compact. Then there exists a closed bounded convex non-weakly compact set $D$ in a Banach space $Y$ such that $D$ is finitely representable in $C$. By James' characterization of non-weakly compact subsets to $D$ (see [12]), there exist $\theta>0$, a sequence $\left\{x_{n}\right\}$ in $D$ and $\left\{x_{n}^{*}\right\}$ in $S_{Y^{*}}$ such that for all $m, n \in \mathbb{N}$,

$$
\left\langle x_{m}^{*}, x_{n}\right\rangle= \begin{cases}2 \theta, & m \leq n, \\ 0, & n<m .\end{cases}
$$

By hypothesis, for every $n \in \mathbb{N}$, the $\left(2^{n}-1\right)$-simplex $S_{2^{n}}:=\operatorname{co}\left\{x_{1}, x_{2}, \ldots, x_{2^{n}}\right\}$ is finitely representable in $C$. Thus, there is a $\left(1+\frac{1}{2}\right)$-affine embedding $T_{n}$ from $S_{2^{n}}$ to $C$. Let

$$
y_{i}=T_{n}\left(x_{i}\right), \quad i=1, \ldots, 2^{n} .
$$

It is easy to see that for $1 \leq k \leq 2^{n}-1$,

$$
\operatorname{dist}\left(\operatorname{co}\left\{y_{1}, \ldots, y_{k}\right\}, \operatorname{co}\left\{y_{k+1}, \ldots, y_{2^{n}}\right\}\right) \geq \theta,
$$

that is, (iii) holds.

(iii) $\Rightarrow$ (ii). Use an argument like that in [13.

(ii) $\Rightarrow$ (i). Suppose that $C$ has the finite tree property. Then applying Lemma 2.13 to $C$, we obtain a (bounded) closed convex set $A$ in a Banach 
space $E$ such that $D$ has the infinite tree property and is finitely representable in $C$. Therefore, $A$ is not weakly compact by James' argument in [14] (see also [2, the 232-235]). This in turn implies that $C$ is not superweakly compact.

Corollary 2.15 .

(i) Every compact convex subset of a Banach space is super-weakly compact.

(ii) A Banach space is super-reflexive if and only if its closed unit ball is super-weakly compact.

(iii) Every bounded convex subset of a super-reflexive Banach space is relatively super-weakly compact.

(iv) A closed convex set is super-weakly compact if and only if every closed separable convex subset of it is super-weakly compact.

3. Super-weakly compact convex sets characterized by $\varepsilon$-uniformly convex functions. In this section, we establish a new geometric characterization of super-weakly compact convex subsets in terms of $\varepsilon$-uniformly convex functions; this characterization implies that $C \times D, C-D$ and $\overline{\mathrm{co}}\{C \cup(-C)\}$ are super-weakly compact whenever $C$ and $D$ are superweakly compact convex subsets. The main idea of this section was motivated by Enflo [9] in the part showing that every super-reflexive space admits an equivalent uniformly convex norm, and by Cheng, Wu, Xue and Yao [3] in discussing equivalence of a space being uniformly convexifiable and existence of uniformly convex functions on some subsets.

Suppose that $C \subset X$ is a nonempty closed bounded convex set with $0 \in C$, and that $\rho$ is the Minkowski functional of $C$. Then $\rho$ is an extended nonnegative-valued and positively homogenous convex function on $X$ with effective domain $\operatorname{dom} \rho \equiv\{x \in X: \rho(x)<\infty\}=\bigcup_{\lambda>0} \lambda C$.

Definition 3.1. For the set $C$ and the function $\rho$ as above, let $\varepsilon>0$ and $z \in \operatorname{dom} \rho$ be given. A pair $\left(x_{1}, x_{2}\right) \in(\operatorname{dom} \rho)^{2}$ is said to be a $(1, \varepsilon, \rho)$ partition of $z$ if

$$
x_{1}+x_{2}=z, \quad \rho\left(x_{1}\right)=\rho\left(x_{2}\right), \quad\left\|\frac{x_{1}}{\rho\left(x_{1}\right)}-\frac{x_{2}}{\rho\left(x_{2}\right)}\right\| \geq \varepsilon .
$$

We denote

$$
\mathscr{P}_{1}(z, \varepsilon)=\left\{\left(x_{1}, x_{2}\right) \in(\operatorname{dom} \rho)^{2}:\left(x_{1}, x_{2}\right) \text { is a }(1, \varepsilon, \rho) \text {-partition of } z\right\} .
$$

We call $\left(x_{1}, x_{2}, x_{3}, x_{4}\right)$ in $(\operatorname{dom} \rho)^{4}$ a $(2, \varepsilon, \rho)$-partition of $z$ if

$$
\left(x_{1}+x_{2}, x_{3}+x_{4}\right) \in \mathscr{P}_{1}(z, \varepsilon)
$$


and

$$
\begin{gathered}
\rho\left(x_{1}\right)=\rho\left(x_{2}\right), \quad \rho\left(x_{3}\right)=\rho\left(x_{4}\right), \\
\left\|\frac{x_{1}}{\rho\left(x_{1}\right)}-\frac{x_{2}}{\rho\left(x_{2}\right)}\right\| \geq \varepsilon, \quad\left\|\frac{x_{3}}{\rho\left(x_{3}\right)}-\frac{x_{4}}{\rho\left(x_{4}\right)}\right\| \geq \varepsilon .
\end{gathered}
$$

We also set

$$
\begin{aligned}
& \mathscr{P}_{2}(z, \varepsilon) \\
& \quad=\left\{\left(x_{1}, x_{2}, x_{3}, x_{4}\right) \in(\operatorname{dom} \rho)^{4}:\left(x_{1}, x_{2}, x_{3}, x_{4}\right) \text { is a }(2, \varepsilon, \rho) \text {-partition of } z\right\} .
\end{aligned}
$$

Inductively, we say that $\left(x_{1}, \ldots, x_{2^{n}}\right) \in(\operatorname{dom} \rho)^{2^{n}}$ is an $(n, \varepsilon, \rho)$-partition of $z$, written $\left(x_{1}, \ldots, x_{2^{n}}\right) \in \mathscr{P}_{n}(z, \varepsilon)$, if

$$
\left(x_{1}+x_{2}, x_{3}+x_{4}, \ldots, x_{2^{n}-1}+x_{2^{n}}\right) \in \mathscr{P}_{n-1}(z, \varepsilon)
$$

and

$$
\rho\left(x_{2 k-1}\right)=\rho\left(x_{2 k}\right), \quad\left\|\frac{x_{2 k-1}}{\rho\left(x_{2 k-1}\right)}-\frac{x_{2 k}}{\rho\left(x_{2 k}\right)}\right\| \geq \varepsilon \quad \text { for } k=1, \ldots, 2^{n-1} .
$$

Lemma 3.2. Suppose that $C$ is a closed bounded convex set in $X$ with $\operatorname{dim}(\operatorname{span} C) \geq 3$. Then there exist $x_{0} \in C$ and $\varepsilon>0$ such that for every nonzero $z \in C_{x_{0}} \equiv \bigcup_{\lambda>0} \lambda\left(C-x_{0}\right)$ and every positive integer $n$, there exists an $(n, \varepsilon, \rho)$-partition of $z$ in $C_{x_{0}}$, where $\rho$ is the Minkowski functional of $C-x_{0}$. In particular, if $C$ is symmetric, then we can put $x_{0}=0$.

Proof. Since $\operatorname{dim}(\operatorname{span} C) \geq 3$, there are affinely independent vectors $\left\{x_{i}\right\}_{i=1}^{4}$ in $C$ such that the 3 -simplex $S_{3}:=\operatorname{co}\left\{x_{i}\right\}_{i=1}^{4}$ has nonempty interior relative to the affine subspace $A \equiv$ aff $\left\{x_{i}\right\}_{i=1}^{4}$ of three dimensions. Choose any $x_{0}$ in the relative interior of $S_{3}$. Take $\delta>0$ such that $B\left(x_{0}, \delta\right) \cap A \subset S_{3}$. Thus, the linear subspace $A_{x_{0}}:=A-x_{0}$ is contained in $C_{x_{0}}$.

Let $\varepsilon=\delta / 3$ and pick $z \in C_{x_{0}} \backslash\{0\}$. Without loss of generality we assume that $\rho(z)=1$. Now, for every $n \in \mathbb{N}$, we want to produce an $(n, \varepsilon, \rho)$-partition of $z$.

Let $L_{z}=\operatorname{span}\left\{S_{3}-x_{0}, z\right\}$ and $S_{z}=\bigcup_{\lambda>0} \lambda \operatorname{co}\left\{S_{3}-x_{0}, z\right\}$. Since $\rho$ is continuous on $S_{z}$, the subdifferential mapping $\partial \rho: S_{z} \rightarrow 2^{L_{z}^{*}}$ is nonemptyvalued everywhere in $S_{z}$. Choose any $x_{1}^{*} \in \partial \rho(z)$. Then $\left\langle x_{1}^{*}, x\right\rangle \leq \rho(x)$ for all $x \in S_{z}$ and $\left\langle x_{1}^{*}, z\right\rangle=\rho(z)$. We extend $x_{1}^{*}$ to the whole space $X$ and still denote it by $x_{1}^{*}$. Then choose $x_{2}^{*} \in X^{*}$ with $\left\|x_{2}^{*}\right\|=1$ such that $\left\langle x_{2}^{*}, z\right\rangle=\|z\|$. Now, put

$$
H_{i}=\left\{x \in X:\left\langle x_{i}^{*}, x\right\rangle=0\right\}, \quad i=1,2 .
$$

Then the set $H_{1} \cap H_{2} \cap\left(S_{3}-x_{0}\right)\left(\subset C-x_{0}\right)$ contains two vectors $\pm u_{1}$ with $\left\|u_{1}\right\| \geq \delta$. We define $f, g:[-1,1] \rightarrow \mathbb{R}$ by

$$
f(\alpha)=\rho\left(z+\left(\alpha z+u_{1}\right)\right), \quad g(\alpha)=\rho\left(z-\left(\alpha z+u_{1}\right)\right)
$$


Note that

$$
\left\langle x_{1}^{*}, z \pm u_{1}\right\rangle=\left\langle x_{1}^{*}, z\right\rangle=\rho(z)=1 .
$$

We have

$$
\begin{aligned}
f(1) & =\rho\left(2 z+u_{1}\right) \geq\left\langle x_{1}^{*}, 2 z+u_{1}\right\rangle=2, \quad f(-1)=\rho\left(u_{1}\right) \leq 1, \\
g(-1) & =\rho\left(2 z-u_{1}\right) \geq\left\langle x_{1}^{*}, 2 z-u_{1}\right\rangle=2, \quad g(1)=\rho\left(-u_{1}\right) \leq 1 .
\end{aligned}
$$

Therefore, there exists $\alpha_{1} \in(-1,1)$ such that $f\left(\alpha_{1}\right)=g\left(\alpha_{1}\right)$, that is,

$$
\rho\left(z+\left(\alpha_{1} z+u_{1}\right)\right)=\rho\left(z-\left(\alpha_{1} z+u_{1}\right)\right) .
$$

Set

$$
z_{1}=\frac{z+\left(\alpha_{1} z+u_{1}\right)}{2}, \quad z_{2}=\frac{z-\left(\alpha_{1} z+u_{1}\right)}{2} .
$$

Then

$$
z_{1}+z_{2}=z, \quad \rho\left(z_{1}\right)=\rho\left(z_{2}\right) \leq 3 / 2
$$

and

$\left\|\frac{z_{1}}{\rho\left(z_{1}\right)}-\frac{z_{2}}{\rho\left(z_{2}\right)}\right\| \geq \frac{2}{3}\left\|\alpha_{1} z+u_{1}\right\| \geq \frac{2}{3} \max \left\{\left|\alpha_{1}\right|\|z\|,\left\|u_{1}\right\|-\left|\alpha_{1}\right|\|z\|\right\} \geq \frac{\delta}{3}=\varepsilon$,

that is, $\left(z_{1}, z_{2}\right)$ is a $(1, \varepsilon, \rho)$-partition of $z$.

Next, let $y_{i}=z_{i} / \rho\left(z_{i}\right), i=1,2$. We substitute $y_{i}$ for $z$ and repeat the procedure above to obtain

$$
\alpha_{1, i} \in(-1,1), \quad \pm u_{1, i} \in C_{x_{0}} \quad \text { with } \quad\left\|u_{1, i}\right\| \geq \delta
$$

and

$$
y_{i, 1}=\frac{y_{i}+\left(\alpha_{1, i} y_{i}+u_{1, i}\right)}{2}, \quad y_{i, 2}=\frac{y_{i}-\left(\alpha_{1, i} y_{i}+u_{1, i}\right)}{2}
$$

for $i=1,2$ such that

$$
y_{i, 1}+y_{i, 2}=y_{i}, \quad \rho\left(y_{i, 1}\right)=\rho\left(y_{i, 2}\right) \leq 3 / 2
$$

and

$$
\left\|\frac{y_{i, 1}}{\rho\left(y_{i, 1}\right)}-\frac{y_{i, 2}}{\rho\left(y_{i, 2}\right)}\right\| \geq \varepsilon \quad \text { for } i=1,2 .
$$

Let $z_{i, j}=\rho\left(z_{i}\right) y_{i, j}, i, j=1,2$. Thus for $i=1,2$,

$$
z_{i}=\left(y_{i, 1}+y_{i, 2}\right) \rho\left(z_{i}\right)=z_{i, 1}+z_{i, 2}, \quad \rho\left(z_{i, 1}\right)=\rho\left(z_{i, 2}\right)
$$

and

$$
\left\|\frac{z_{i, 1}}{\rho\left(z_{i, 1}\right)}-\frac{z_{i, 2}}{\rho\left(z_{i, 2}\right)}\right\|=\left\|\frac{y_{i, 1}}{\rho\left(y_{i, 1}\right)}-\frac{y_{i, 2}}{\rho\left(y_{i, 2}\right)}\right\| \geq \varepsilon .
$$

This says that $\left(z_{1,1}, z_{1,2}, z_{2,1}, z_{2,2}\right)$ is a $(2, \varepsilon, \rho)$-partition of $z$.

Inductively, for every $n \in \mathbb{N}$, we can obtain an $(n, \varepsilon, \rho)$-partition $\left(z_{\varepsilon_{1}, \ldots, \varepsilon_{n}}\right.$ : $\varepsilon_{i}=1,2$ and $\left.i=1, \ldots, n\right)$ of $z$.

Next, we make full use of Enflo's technique of construction of a uniformly convex norm from absence of finite trees. We only sketch the proofs of the 
following three lemmas; the details can be completed without much effort by consultling Enflo's proofs of the corresponding lemmas in [9].

Lemma 3.3. Let the sets $C, C_{x_{0}}, \varepsilon>0$ and the Minkowski functional $\rho$ be as in Lemma 3.2. Assume that $C$ is super-weakly compact. Then there exist $0<\delta<\varepsilon$ and $n \in \mathbb{N}$ such that for all $z \in C_{x_{0}}$ and all $(n, \varepsilon, \rho)$-partitions $\left(x_{1}, \ldots, x_{2^{n}}\right)$ of $z$, the following inequality holds:

$$
\sum_{i=1}^{2^{n}} \rho\left(x_{i}\right) \geq(1+\delta) \rho(z)
$$

Proof (sketch). Suppose, to the contrary, that for every $n \in \mathbb{N}$ and $\delta=$ $2^{-n}$, there exist $z_{0} \in C_{x_{0}}$ and an $(n, \varepsilon, \rho)$-partition $\left(x_{1}, \ldots, x_{2^{n}}\right)$ of $z_{0}$ such that

$$
\sum_{i=1}^{2^{n}} \rho\left(x_{i}\right)<\left(1+2^{-n}\right) \rho\left(z_{0}\right) \text {. }
$$

We assume again that $\rho\left(z_{0}\right)=1$. Let

$$
A_{n}=\left\{x_{j}^{(k)}=2^{k} \sum_{i=2^{n-k}(j-1)+1}^{2^{n-k} j} x_{i}: k=1, \ldots, n \text { and } j=1, \ldots, 2^{k}\right\} .
$$

Then we see that $A_{n} \subset 2\left(C-x_{0}\right)$ and $A_{n}$ is an $(n, \varepsilon)$-tree. This contradicts the assumption that $C$ is super-weakly compact.

Lemma 3.4. Suppose that $C$ is a nonempty super-weakly compact set. Then there exist $x_{0} \in C$ and $\varepsilon_{0}>0$ with the property that for every $0<\varepsilon$ $<\varepsilon_{0}$, there exist a function $f$ on $C_{x_{0}}$ and $0<\gamma<\min \{1 / 8, \varepsilon /(1+\varepsilon)\}$ such that:

(i) $f(x) \geq 0, f(\alpha x)=\alpha f(x)$ for all $\alpha \geq 0$, and $f(x)=0 \Leftrightarrow x=0$.

(ii) $(1-\gamma) \rho(x) \leq f(x) \leq(1-\gamma / 3) \rho(x)$, where $\rho$ denotes the Minkowski functional of $C-x_{0}$.

(iii) There exists $\delta>0$ such that $\rho(x)=\rho(y)=1$ and $\|x-y\| \geq \varepsilon$ imply $f(x+y)<f(x)+f(y)-\delta$.

In particular:

(iv) If $C$ is symmetric, then we can put $x_{0}=0$. Therefore $\rho$ is a lower semi-continuous norm on $\operatorname{span} C$ and there exists a function $f$ such that (i)-(iii) hold on $\operatorname{span} C$.

Proof. It is easy to observe that these assertions are true for $\operatorname{dim}(\operatorname{span} C)$ $<\infty$. Therefore, it suffices to consider the case $\operatorname{dim}(\operatorname{span} C)=\infty$.

By Lemma 3.2, there exist $x_{0} \in C$ and $\varepsilon_{0}>0$ such that for every $n \in \mathbb{N}$, $z \in C_{x_{0}}$ and $0<\varepsilon \leq \varepsilon_{0}$, there is an $(n, \varepsilon, \rho)$-partition of $z$ in $C_{x_{0}}$. In view 
of Lemma 3.3, there exist $0<\gamma<\min \{1 / 8, \varepsilon /(1+\varepsilon)\}$ and $n \in \mathbb{N}$ such that for all $z \in C_{x_{0}}$ and all $(n, \varepsilon, \rho)$-partitions $\left(x_{1}, \ldots, x_{2^{n}}\right)$ of $z$,

$$
\sum_{i=1}^{2^{n}} \rho\left(x_{i}\right) \geq(1+\gamma) \rho(z) .
$$

Now, we fix such $z, \varepsilon, n, \gamma$ and let

$$
\begin{aligned}
f(z)=\inf \left\{\sum_{i=1}^{2^{m}} \frac{\rho\left(x_{i}\right)}{1+\frac{\gamma}{2}\left(1+\frac{1}{4}+\ldots+\frac{1}{4^{m}}\right)}: 0 \leq m \leq n\right. \text { and } \\
\left.\left(x_{1}, \ldots, x_{2^{m}}\right) \text { is an }(m, \varepsilon, \rho) \text {-partition of } z\right\} .
\end{aligned}
$$

Clearly, $f$ satisfies (i) and (ii). It follows from (3.2) and (3.3) that we can assume $0 \leq m \leq n-1$. Suppose $x, y \in C_{x_{0}}$ with $\rho(x)=\rho(y)=1$ and with $\|x-y\| \geq \varepsilon$. Then

$$
f(x)+f(y)-f(x+y) \geq \frac{\gamma}{2} \cdot \frac{1}{4^{k+3}} \geq \frac{\gamma}{2} \cdot \frac{1}{4^{n+2}}
$$

whenever $0<\gamma<1 / 8$. We finish the proof by taking $\delta=\frac{\gamma}{2} \cdot 4^{-(n+2)}$.

Lemma 3.5. Suppose that $C$ is a nonempty super-weakly compact convex set in $X$. Then there exist $\varepsilon_{0}>0$ and $x_{0} \in C$ such that for every $0<\varepsilon<\varepsilon_{0}$, there are $0<\gamma<\min \{1 / 8, \varepsilon /(1+\varepsilon)\}, \delta>0$ and a Minkowski functional $p_{\varepsilon}$ with $\operatorname{dom} p_{\varepsilon}=C_{x_{0}}$ such that:

(i) $(1-\gamma) \rho(x) \leq p_{\varepsilon}(x) \leq(1-\gamma / 3) \rho(x)$, where $\rho$ is the Minkowski functional of $C-x_{0}$.

(ii) $\rho(x)=\rho(y)=1$ and $\|x-y\| \geq \varepsilon$ imply $p_{\varepsilon}(x+y) \leq p_{\varepsilon}(x)+p_{\varepsilon}(y)-\varepsilon \delta$.

(iii) For every $0<\beta \leq 1$, if $\rho\left(x_{n}\right) \rightarrow \beta, \rho\left(y_{n}\right) \rightarrow \beta$ and $p_{\varepsilon}\left(x_{n}\right)+p_{\varepsilon}\left(y_{n}\right)-$ $p_{\varepsilon}\left(x_{n}+y_{n}\right) \rightarrow 0$ then $\lim _{n \rightarrow \infty}\left\|x_{n}-y_{n}\right\|<\beta \varepsilon$.

In particular:

(iv) If $C$ is symmetric, then we can put $x_{0}=0$. Therefore $\rho$ is a lower semi-continuous norm on $Y \equiv \operatorname{span} C$ and there exists a norm $p_{\varepsilon}$ on span $C$ satisfying (i)-(iii).

Proof. By Lemma 3.4, there exist $x_{0} \in C$ and $\varepsilon_{0}>0$ such that for every $0<5 \varepsilon_{1} \equiv \varepsilon<\varepsilon_{0}$, there are a nonnegative real-valued positively homogenous function $f$ on $C_{x_{0}}$ and $0<\gamma<\min \left\{1 / 8, \varepsilon_{1} /\left(1+\varepsilon_{1}\right)\right\}$ satisfying:

(a) $(1-\gamma) \rho(x) \leq f(x) \leq(1-\gamma / 3) \rho(x)$ for all $x \in C_{x_{0}}$.

(b) There exists $\delta_{1}>0$ such that $\rho(x)=\rho(y)=1$ and $\|x-y\| \geq \varepsilon_{1}$ imply $f(x+y)<f(x)+f(y)-\delta_{1}$. 
Without loss of generality, we can assume $C-x_{0} \subset B_{X}$. Then $\|\cdot\| \leq \rho$ on $X$. Now, let

$$
p_{\varepsilon}(x)= \begin{cases}\inf \left\{\sum_{i=1}^{n} f\left(x_{i}\right): n \in \mathbb{N}, x_{i} \in C_{x_{0}} \text { with } \sum_{i=1}^{n} x_{i}=x\right\} & \text { if } x \in C_{x_{0}}, \\ +\infty & \text { otherwise. }\end{cases}
$$

Clearly, $p_{\varepsilon}$ is an extended nonnegative-valued sublinear function (hence, a Minkowski functional) on $X$ with $\operatorname{dom} p_{\varepsilon}=C_{x_{0}}$. Then (i)-(iv) can be deduces using Enflo's procedure from [9].

Definition 3.6. Suppose $f$ is a convex function on a nonempty convex set $C$ in a Banach space $X$ and $\varepsilon>0$. Then:

(i) $f$ is called $\varepsilon$-uniformly convex on $C$ if there exists $\delta>0$ such that $x, y \in C$ with $\|x-y\| \geq \varepsilon$ implies $\frac{1}{2}[f(x)+f(y)]-f\left(\frac{x+y}{2}\right)>\delta$.

(ii) $f$ is said to be uniformly convex on $C$ if $f$ is $\varepsilon$-uniformly convex for all $\varepsilon>0$.

TheOREM 3.7. Suppose that $C$ is a nonempty super-weakly compact convex set in a Banach space $X$. Then there exists $\varepsilon_{0}>0$ such that for every $0<\varepsilon<\varepsilon_{0}$, there is a bounded $\varepsilon$-uniformly convex function on $C$.

Proof. By Lemma 3.5, we can find $x_{0} \in C$ and $\varepsilon_{0}>0$ such that for every $0<\varepsilon<\varepsilon_{0}$, there exist $\delta>0$ and a Minkowski functional $p_{\varepsilon}$ with $\operatorname{dom} p_{\varepsilon}=C_{x_{0}}$ which is bounded on $C-x_{0}$ and satisfies

$$
p_{\varepsilon}(x+y) \leq p_{\varepsilon}(x)+p_{\varepsilon}(y)-\varepsilon \delta
$$

whenever $\rho(x)=\rho(y)=1$ and $\|x-y\| \geq \varepsilon / 2$, where $\rho$ still denotes the Minkowski functional of $C-x_{0}$.

The function $f=p_{\varepsilon}^{2}(\cdot)+\rho^{2}(\cdot)$ is convex as a sum of such functions. Now we claim that $g:=f\left(\cdot-x_{0}\right)$ is $\varepsilon$-uniformly convex on $C$. Obviously, it suffices to show that $f$ is $\varepsilon$-uniformly convex on $C-x_{0}$.

Assume that $f$ is not $\varepsilon$-uniformly convex. Then there are two sequences $\left\{x_{n}\right\}$ and $\left\{y_{n}\right\}$ in $C-x_{0}$ with $\left\|x_{n}-y_{n}\right\| \geq \varepsilon$ such that

$$
\frac{1}{2}\left[f\left(x_{n}\right)+f\left(y_{n}\right)\right]-f\left(\frac{x_{n}+y_{n}}{2}\right) \rightarrow 0,
$$

which in turn implies that

$$
p_{\varepsilon}\left(x_{n}\right)-p_{\varepsilon}\left(y_{n}\right) \rightarrow 0, \quad \rho\left(x_{n}\right)-\rho\left(y_{n}\right) \rightarrow 0
$$

and

$$
p_{\varepsilon}\left(\frac{x_{n}+y_{n}}{2}\right)-\frac{1}{2}\left[p_{\varepsilon}\left(x_{n}\right)+p_{\varepsilon}\left(y_{n}\right)\right] \rightarrow 0 .
$$

We can assume that

$$
\rho\left(x_{n}\right) \rightarrow r, \quad \rho\left(y_{n}\right) \rightarrow r
$$


for some $0<r \leq 1$. Let $x_{n}^{\prime}=\left(r / \rho\left(x_{n}\right)\right) x_{n}$ and $y_{n}^{\prime}=\left(r / \rho\left(y_{n}\right)\right) y_{n}$ for all $n \in \mathbb{N}$. Then it is not difficult to show

$$
p_{\varepsilon}\left(\frac{x_{n}^{\prime}+y_{n}^{\prime}}{2}\right)-p_{\varepsilon}\left(\frac{x_{n}+y_{n}}{2}\right) \rightarrow 0 .
$$

Therefore

$$
p_{\varepsilon}\left(\frac{u_{n}+v_{n}}{2}\right)-\frac{1}{2}\left[p_{\varepsilon}\left(u_{n}\right)+p_{\varepsilon}\left(v_{n}\right)\right] \rightarrow 0
$$

where $u_{n}=(1 / r) x_{n}^{\prime}$ and $v_{n}=(1 / r) y_{n}^{\prime}$ satisfy $\rho\left(u_{n}\right)=\rho\left(v_{n}\right)=1$. Applying Lemma 3.5(iii), we obtain $\varlimsup_{n \rightarrow \infty}\left\|u_{n}-v_{n}\right\|<\varepsilon$. On the other hand,

$$
\varlimsup_{n \rightarrow \infty}\left\|u_{n}-v_{n}\right\|=\varlimsup_{n \rightarrow \infty} \frac{1}{r}\left\|x_{n}^{\prime}-y_{n}^{\prime}\right\|=\varlimsup_{n \rightarrow \infty} \frac{1}{r}\left\|x_{n}-y_{n}\right\| \geq \frac{\varepsilon}{r} \geq \varepsilon,
$$

and this is a contradiction.

THeOrem 3.8. Suppose that $C$ is a nonempty bounded closed convex set in $X$. Assume that there is $\varepsilon_{0}>0$ such that for every $0<\varepsilon<\varepsilon_{0}$ there exists a bounded $\varepsilon$-uniformly convex function on $C$. Then $C$ is super-weakly compact. Therefore, every bounded closed uniformly convexifiable set is super-weakly compact.

Proof. Suppose that $C$ is not super-weakly compact. Then by Theorem 2.14, there exists $\theta>0$ such that for every $n \in \mathbb{N}$, there exist $x_{i} \in C$ for $i=1,2, \ldots, 2^{n}$ satisfying

$$
\operatorname{dist}\left(\operatorname{co}\left\{x_{1}, \ldots, x_{k}\right\}, \operatorname{co}\left\{x_{k+1}, \ldots, x_{2^{n}}\right\}\right)>\theta
$$

for all $1 \leq k<2^{n}$. Clearly, $\operatorname{dim}(\operatorname{span} C)=\infty$. Now, fix any $0<\varepsilon<$ $\min \left\{\theta, \varepsilon_{0}\right\}$, and let $f$ be a bounded $\varepsilon$-uniformly convex function on $C$, with $\delta>0$ witnessing that. Let $-\infty<\alpha=\inf _{C} f$ and $\sup _{C} f=\beta<\infty$. Since $\left\|x_{i}-x_{j}\right\| \geq \theta>\varepsilon$ for all $1 \leq i \neq j \leq 2^{n}$, we know that for all $1 \leq i \neq j \leq 2^{n}$,

$$
f\left(\frac{x_{i}+x_{j}}{2}\right)<\frac{1}{2}\left(f\left(x_{i}\right)+f\left(x_{j}\right)\right)-\delta .
$$

Note that $\left\|\frac{x_{1}+x_{2}}{2}-\frac{x_{3}+x_{4}}{2}\right\|>\varepsilon$. We see that

$$
\begin{aligned}
f\left(\frac{x_{1}+x_{2}+x_{3}+x_{4}}{4}\right) & <\frac{1}{2}\left(f\left(\frac{x_{1}+x_{2}}{2}\right)+f\left(\frac{x_{3}+x_{4}}{2}\right)\right)-\delta \\
& <\frac{1}{4}\left(f\left(x_{1}\right)+f\left(x_{2}\right)+f\left(x_{3}\right)+f\left(x_{4}\right)\right)-2 \delta .
\end{aligned}
$$

Inductively, for all positive integers $n$,

$$
-\infty<\alpha \leq f\left(\frac{\sum_{i=1}^{2^{n}} x_{i}}{2^{n}}\right) \leq \frac{1}{2^{n}}\left(\sum_{i=1}^{2^{n}} f\left(x_{i}\right)\right)-n \delta \leq \beta-n \delta,
$$

and this is a contradiction. 
Putting together Theorems 3.7 and 3.8, we have

Theorem 3.9. A nonempty closed bounded convex set $C$ in $X$ is superweakly compact if and only if for every $\varepsilon>0$, there exists a bounded $\varepsilon$ uniformly convex function on $C$.

The following is a consequence of Theorem 2.14.

Proposition 3.10. Suppose $X$ and $Y$ are Banach spaces and $C \subset X$ is a super-weakly compact convex set. Let $T: C \rightarrow Y$ be a uniformly continuous affine mapping. Then $T C$ is relatively super-weakly compact in $Y$.

Corollary 3.11 . Suppose that $X$ and $Y$ are Banach spaces and $B, C \subset$ $X$ and $D \subset Y$ are super-weakly compact convex sets. Then:

(i) $C \times D$ is super-weakly compact in $X \times Y$.

(ii) $B-C$ is super-weakly compact in $X$.

(iii) $\overline{\mathrm{co}}\{C \cup(-C)\}$ is super-weakly compact in $X$.

Proof. (i) We equip $X \times Y$ with the max-norm, i.e.

$$
\|(x, y)\|=\max \{\|x\|,\|y\|\} \quad \text { for all }(x, y) \in X \times Y .
$$

Let $f$ be a bounded $\varepsilon$-uniformly convex function on $A$ and $g$ a bounded $\varepsilon$ uniformly convex function on $B$. It is easy to see that $h(x, y)=f(x)+g(y)$ is again a bounded $\varepsilon$-uniformly convex function on $A \times B$. By Theorem 3.8, $A \times B$ is super-weakly compact.

(ii) By Proposition 3.10 and (i) just proved, it suffices to note that $T$ : $C \times D \rightarrow X$ defined by $T(x, y)=x-y$ is a uniformly continuous affine mapping.

(iii) Again we can use Proposition 3.10 and (i), because $\operatorname{co}\{W \cup-W\}$ is the image of the super-weakly compact convex set $W \times(-W) \times[0,1]$ under the affine mapping $T: X^{2} \times[0,1] \rightarrow X$ defined by $T(x, y, \lambda)=\lambda x+(1-\lambda) y$.

\section{Every super-weakly compact convex set is uniformly convexi-}

fiable. We have shown in Section 3 that a nonempty closed bounded convex set $C$ of a Banach space $(X,\|\cdot\|)$ is super-weakly compact (if and only) if for every $\varepsilon>0$ there exists a bounded $\varepsilon$-uniformly convex function on $C$, in particular, every closed bounded uniformly convexifiable convex set is super-weakly compact. In this section, we will show that the converse is also true, i.e. every super-weakly compact convex set is uniformly convexifiable.

Recall that a convex set $C$ of a Banach space $X$ is said to be uniformly convex (with respect to the original norm $\|\cdot\|$ ) if $f=\left\|\cdot-x_{0}\right\|^{2}$ is a uniformly convex function on $C$; in this case, we also say that $\|\cdot\|$ is uniformly convex on $C$. The set $C$ is said to be uniformly convexifiable provided there exists an equivalent norm $|\cdot|$ on $X$ that is uniformly convex on $C$, or equivalently, 
$2\left(\left|x_{n}\right|^{2}+\left|y_{n}\right|^{2}\right)-\left|x_{n}+y_{n}\right|^{2} \rightarrow 0$ implies $\left|x_{n}-y_{n}\right| \rightarrow 0$ whenever $\left\{x_{n}\right\},\left\{y_{n}\right\} \subset$ $C-x_{0}$ for any fixed $x_{0} \in C$.

For a Minkowski functional $\rho$ on $X$, we denote by $B_{\rho}(r)$ the level set $\{x \in X: \rho(x) \leq r\}$.

It is easy to show the following assertions.

Proposition 4.1. Suppose that $C$ is a nonempty convex set in a Banach $\operatorname{space}(X,\|\cdot\|)$. Then:

(i) $C$ is uniformly convex if and only if for every $r>0$ and every $x_{0} \in C$,

$$
\left\|x_{n}+y_{n}\right\| \rightarrow 2 r \quad \text { implies }\left\|x_{n}-y_{n}\right\| \rightarrow 0
$$

whenever $\left\{x_{n}\right\},\left\{y_{n}\right\}$ are two sequences in $B_{\|\cdot\|}(r) \cap\left(C-x_{0}\right)$.

(ii) $X$ is uniformly convex if $B_{X}$ is uniformly convex.

Proposition 4.2. Suppose that $C$ is a symmetric convex set in the space $X$. Then the following are equivalent:

(i) $C$ is uniformly convex.

(ii) For every $r>0,\left\|x_{n}+y_{n}\right\| \rightarrow 2 r$ implies $\left\|x_{n}-y_{n}\right\| \rightarrow 0$ whenever $\left\{x_{n}\right\},\left\{y_{n}\right\}$ are two sequences in $B_{\|\cdot\|}(r) \cap C$.

LEMma 4.3. Assume that $C$ is a super-weakly compact and convex symmetric set in a Banach space $(X,\|\cdot\|)$. Then there is $\varepsilon_{0}>0$ such that for every $0<\varepsilon<\varepsilon_{0}$, there exists a norm $|\cdot|$ on $Y \equiv \operatorname{span} C$ satisfying:

(i) $(1-\varepsilon) \rho(x) \leq|x| \leq \rho(x)$, where $\rho$ denotes the norm (Minkowski functional) generated by $C$.

(ii) $\left|x_{n}\right|+\left|y_{n}\right|-\left|x_{n}+y_{n}\right| \rightarrow 0$ implies $\left\|x_{n}-y_{n}\right\| \rightarrow 0$ whenever $x_{n}, y_{n} \in B_{\rho}$ with $\rho\left(x_{n}\right) \rightarrow 1$ and $\rho\left(y_{n}\right) \rightarrow 1$.

Proof. By Lemma 3.5, there exists $\varepsilon_{0}>0$ such that for every $0<\varepsilon<\varepsilon_{0}$, there are $0<\gamma<\min \{1 / 8, \varepsilon /(1+\varepsilon)\}, \delta>0$ and a norm $p_{\varepsilon}$ on $Y$ satisfying:

(a) $(1-\gamma) \rho(x) \leq p_{\varepsilon}(x) \leq(1-\gamma / 3) \rho(x)$.

(b) For each $0<r \leq 1$ and sequences $\left\{x_{n}\right\},\left\{y_{n}\right\} \subset B_{\rho}(r)$ with $\rho\left(x_{n}\right) \rightarrow r$, $\rho\left(y_{n}\right) \rightarrow r$, we have $\overline{\lim }_{n \rightarrow \infty}\left\|x_{n}-y_{n}\right\|<r \varepsilon$ whenever $p_{\varepsilon}\left(x_{n}+y_{n}\right)-$ $p_{\varepsilon}\left(x_{n}\right)-p_{\varepsilon}\left(y_{n}\right) \rightarrow 0$.

Let $\varepsilon_{n}=\varepsilon / 2^{n}$ and $p_{n} \equiv p_{\varepsilon_{n}}$ for $n=1,2, \ldots$ Finally, let

$$
|x|=\sum_{n=1}^{\infty} 2^{-n} p_{n}(x), \quad x \in Y .
$$

Clearly, $|\cdot|$ is a norm on $Y$ satisfying $(1-\varepsilon) \rho(x) \leq|x| \leq \rho(x)$. We want to show that $|\cdot|$ has the desired properties.

Suppose that $\left\{x_{m}\right\},\left\{y_{m}\right\} \subset B_{\rho}(r)$ with $\rho\left(x_{m}\right) \rightarrow r, \rho\left(y_{m}\right) \rightarrow r$ and $\left|x_{m}\right|+\left|y_{m}\right|-\left|x_{m}+y_{m}\right| \rightarrow 0$. We claim that $\left\|x_{m}-y_{m}\right\| \rightarrow 0$. Without loss of generality we can assume that $r=1$. Suppose, to the contrary, that there 
exist $a>0$ and a subsequence of $\left\{x_{m}-y_{m}\right\}$ still denoted by $\left\{x_{m}-y_{m}\right\}$, such that for all sufficiently large $m \in \mathbb{N},\left\|x_{m}-y_{m}\right\|>a$. Choose $j \in \mathbb{N}$ with $\varepsilon_{j}<a$. Note that

$$
\left|x_{m}\right|+\left|y_{m}\right|-\left|x_{m}+y_{m}\right| \rightarrow 0
$$

implies that for all $i \in \mathbb{N}$,

$$
p_{i}\left(x_{m}\right)+p_{i}\left(y_{m}\right)-p_{i}\left(x_{m}+y_{m}\right) \rightarrow 0 .
$$

Thus, by Lemma 3.5(iii),

$$
\varlimsup_{n \rightarrow \infty}\left\|x_{m}-y_{m}\right\|<\varepsilon_{j}<a .
$$

This is a contradiction.

Lemma 4.4 Suppose that $\|\cdot\|_{1}$ and $\|\cdot\|_{2}$ are norms on a linear space $Y$, and $C \subset Y$ is a convex symmetric set. Then $\|\cdot\|_{2}$ is uniformly convex on $C$ if the following conditions are satisfied:

(i) $\|x\|_{2} \leq\|x\|_{1} \leq 2\|x\|_{2}$ for all $x \in Y$.

(ii) For every $r>0,\left\|x_{n}\right\|_{2}+\left\|y_{n}\right\|_{2}-\left\|x_{n}+y_{n}\right\|_{2} \rightarrow 0$ implies $\left\|x_{n}-y_{n}\right\|_{1}$ $\rightarrow 0$ whenever $x_{n}, y_{n} \in B_{\|\cdot\|_{1}}(r) \cap C$ with $\left\|x_{n}\right\|_{1} \rightarrow r$ and $\left\|y_{n}\right\|_{1} \rightarrow r$.

Proof. For every $r>0$, suppose that $x_{n}, y_{n} \in B_{\|\cdot\|_{2}}(r) \cap C$ are such that $\left\|x_{n}+y_{n}\right\|_{2} \rightarrow 2 r$. We claim that $\left\|x_{n}-y_{n}\right\|_{2} \rightarrow 0$. Indeed, let $\left\{\alpha_{n}\right\},\left\{\beta_{n}\right\} \subset$ $[1 / 2,1]$ be sequences with $\left\|\alpha_{n} x_{n}\right\|_{1}=\left\|x_{n}\right\|_{2}$ and $\left\|\beta_{n} y_{n}\right\|_{1}=\left\|y_{n}\right\|_{2}$. Then it is easy to see that $\alpha_{n} x_{n}, \beta_{n} y_{n} \in C$ and

$\left\|x_{n}+y_{n}\right\|_{2}-\left(\left\|x_{n}\right\|_{2}+\left\|y_{n}\right\|_{2}\right) \leq\left\|\alpha_{n} x_{n}+\beta_{n} y_{n}\right\|_{2}-\left(\left\|\alpha_{n} x_{n}\right\|_{2}+\left\|\beta_{n} y_{n}\right\|_{2}\right) \leq 0$.

Therefore, it follows from $\left\|x_{n}\right\|_{2}+\left\|y_{n}\right\|_{2}-\left\|x_{n}+y_{n}\right\|_{2} \rightarrow 0$ that

$$
\left\|\alpha_{n} x_{n}\right\|_{2}+\left\|\beta_{n} y_{n}\right\|_{2}-\left\|\alpha_{n} x_{n}+\beta_{n} y_{n}\right\|_{2} \rightarrow 0 .
$$

By the assumption of the lemma, we have

$$
\left\|\alpha_{n} x_{n}-\beta_{n} y_{n}\right\|_{1} \rightarrow 0 .
$$

Hence

$$
\begin{aligned}
r \lim _{n \rightarrow \infty}\left|\alpha_{n}-\beta_{n}\right| & =\lim _{n \rightarrow \infty}\left|\left\|\alpha_{n} x_{n}\right\|_{2}-\left\|\beta_{n} y_{n}\right\|_{2}\right| \leq \lim _{n \rightarrow \infty}\left\|\alpha_{n} x_{n}-\beta_{n} y_{n}\right\|_{2} \\
& \leq \lim _{n \rightarrow \infty}\left\|\alpha_{n} x_{n}-\beta_{n} y_{n}\right\|_{1}=0
\end{aligned}
$$

and note that

$$
\frac{1}{2}\left\|x_{n}-\gamma_{n} y_{n}\right\|_{2} \leq\left\|\alpha_{n} x_{n}-\beta_{n} y_{n}\right\|_{2} \leq\left\|\alpha_{n} x_{n}-\beta_{n} y_{n}\right\|_{1}
$$

where $\gamma_{n}=\beta_{n} / \alpha_{n}$. Thus $\left\|x_{n}-y_{n}\right\|_{2} \rightarrow 0$.

In the following, we show that an analogue of Grothendieck's Lemma for super-weakly compact convex sets is still valid. 
Lemma 4.5. A nonempty closed convex set $C$ in a Banach space $X$ is super-weakly compact (if and only) if for every $\varepsilon>0$ there exists a superweakly compact convex set $C_{\varepsilon}$ in $X$ such that $C \subset C_{\varepsilon}+\varepsilon B_{X}$.

Proof. It suffices to show sufficiency. Suppose that $C$ is not super-weakly compact. By Theorem 2.14, we can find $0<\theta<1$ such that for every $n \in \mathbb{N}$, there exist $x_{i} \in C$ for $i=1, \ldots, n$ such that for every $1 \leq k<n$,

$$
\operatorname{dist}\left(\operatorname{co}\left\{x_{1}, \ldots, x_{k}\right\}, \operatorname{co}\left\{x_{k+1}, \ldots, x_{n}\right\}\right)>\theta \text {. }
$$

Let $\varepsilon=\theta / 4$. There is a super-weakly compact convex set $C_{\varepsilon}$ such that $C \subset C_{\varepsilon}+\varepsilon B_{X}$. Let

$$
x_{i}=y_{i}+z_{i}, \quad i=1, \ldots, n,
$$

where $y_{i} \in C_{\varepsilon}$ and $\left\|z_{i}\right\| \leq \varepsilon$. One checks easily that for every $1 \leq k<n$,

$$
\operatorname{dist}\left(\operatorname{co}\left\{y_{1}, \ldots, y_{k}\right\}, \operatorname{co}\left\{y_{k+1}, \ldots, y_{n}\right\}\right) \geq \varepsilon \text {. }
$$

By Theorem 2.14 again, $C_{\varepsilon}$ is not super-weakly compact, a contradiction.

The following result is the so called Davis-Figiel-Johnson-Pełczyński Lemma (see [8, pp. 250-251]).

Lemma $4.6([5])$. Suppose that $(X,\|\cdot\|)$ is a Banach space with closed unit ball $B_{X}$. Let $W$ be a bounded convex symmetric set in $X$. For each positive integer $n$, let $U_{n}=2^{n} W+2^{-n} B_{X}$. Denote by $\|\cdot\|_{n}$ the Minkowski functional of $U_{n}$, i.e.

$$
\|x\|_{n}=\inf \left\{\alpha>0: x \in \alpha U_{n}\right\}
$$

For $x \in X$, let $\||x|\|$ be given by $\||x|\|=\left(\sum_{n=1}^{\infty}\|x\|_{n}^{2}\right)^{1 / 2}$ and let $Y=\{x \in X$ : $\||| x \mid\|<\infty\}$. Denote by $C$ the $\||\cdot|\|$-closed unit ball of $Y$. Let $J: Y \rightarrow X$ be the natural inclusion. Then:

(i) $W \subset C$.

(ii) $(Y,|||\cdot|||)$ is a Banach space and $J$ is continuous.

(iii) $J^{* *}: Y^{* *} \rightarrow X^{* *}$ is one-to-one and $Y=\left(J^{* *}\right)^{-1}(X)$.

(iv) $(Y,|||\cdot|||)$ is reflexive if (and only if) $W$ is relatively weakly compact in $X$.

This lemma is also called the factorization theorem. Here we need a refined version of the lemma.

Lemma 4.7. Let the Banach spaces $X$ and $Y$, the subsets $W$ and $C$, and the norms $\|\cdot\|,\||\cdot|\|$ and $\|\cdot\|_{n}(n=1,2, \ldots)$ be as in the previous lemma. Then:

(i) The topologies generated by $\|\cdot\|$ and $|\|\cdot \mid\|$ coincide on $W$.

(ii) $(Y,|\|\cdot \mid\|)$ is separable if (and only if) $(Y,\|\cdot\|)$ is separable.

(iii) Every relatively weakly compact set in $X$ is uniformly isomorphic to a weakly compact subset of a reflexive space. 
(iv) If $W$ is relatively super-weakly compact in $(X,\|\cdot\|)$, then $C$ is also super-weakly compact in $(X,\|\cdot\|)$.

Proof. (i) For every positive integer $m$, let

$$
p_{m}(x)=\left(\sum_{n=1}^{m}\|x\|_{n}^{2}\right)^{1 / 2} \quad \text { for } x \in Y .
$$

Then $p_{m}$ is uniformly $\|\cdot\|$-continuous, since every $\|\cdot\|_{n}$ is uniformly $\|\cdot\|$ continuous on $X$. Note that $\|x\|_{n}<2^{-n}$ for every positive integer $n$ and $x \in W$. We know that $p_{m}$ uniformly converges to \|\|$\cdot \| \mid$ on $W$. Putting this together, we see that for every $\varepsilon>0$ there is $\delta>0$ such that, whenever $u, v \in W$ and $\|u-v\|<\delta$, then $\|\mid u-v\| \|<\varepsilon$. And since $\|\cdot\| \leq c \mid\|\cdot\| \|$ with a suitable $c>0$, (i) is proved.

(ii) It is trivial to see that $(Y,\|\cdot\|)$ is separable if $(Y,\||\cdot|\|)$ is separable. Conversely, if $(Y,\|\cdot\|)$ is separable, then $\left(Y,\|\cdot\|_{n}\right)$ is also separable for every positive integer $n$ by noting $\|\cdot\|$ and $\|\cdot\|_{n}$ are equivalent on $Y$. Therefore the direct $\operatorname{sum} \sum_{n=1}^{\infty} \oplus\left(Y,\|\cdot\|_{n}\right)$, equipped with the norm $\left\|\left|\left(x_{n}\right) \|\right|=\left(\sum_{n=1}^{\infty}\left\|x_{n}\right\|_{n}^{2}\right)^{1 / 2}\right.$, is a separable space. We complete the proof of (ii) by observing that $(Y,|\|\cdot\|| \|)$ is isometric to a subspace of $\sum_{n=1}^{\infty} \oplus\left(Y,\|\cdot\|_{n}\right)$.

(iii) This is just a direct consequence of (i), since a subset $K$ is relatively weakly compact if and only if the closed convex hull of $K \cup-K$ is weakly compact.

(iv) It suffices to note that $C \subset 2^{n} W+2^{-n} B_{X}$ for every $n \in \mathbb{N}$ and then apply Lemma 4.5 .

THEOREM 4.8. Suppose that $W$ is a nonempty convex symmetric superweakly compact subset of a Banach space $(X,\|\cdot\|)$. Then there exists a reflexive Banach space $\left(E,\|\cdot\|_{1}\right)$ such that:

(i) $W \subset B_{E} \subset X$.

(ii) The topology of $\|\cdot\|_{1}$ is stronger than that of $\|\cdot\|$ on $E$.

(iii) The topologies generated by $\|\cdot\|$ and $\|\cdot\|_{1}$ coincide on $W$, and $\|\cdot\|_{1}$ is uniformly convex on $W$.

(iv) $\|\cdot\|_{1}$ is uniformly convex with respect to $\|\cdot\|$ in the sense that for any $\|\cdot\|_{1}$-bounded sequences $\left\{x_{n}\right\}$ and $\left\{y_{n}\right\}$ in $E$,

$$
2\left(\left\|x_{n}\right\|_{1}^{2}+\left\|y_{n}\right\|_{1}^{2}\right)-\left\|x_{n}+y_{n}\right\|_{1}^{2} \rightarrow 0 \quad \text { implies } \quad\left\|x_{n}-y_{n}\right\| \rightarrow 0 .
$$

Proof. Applying Lemmas 4.6 and 4.7 for the super-weakly compact set $W$, we produce a reflexive Banach space $(Y,|\|\cdot|\||)$ such that the closed unit ball $C$ of $Y$ is also super-weakly compact in $(X,\|\cdot\|)$ and $W \subset C$. For the convex symmetric super-weakly compact subset $C$ of $X$, we note that the norm $\||\cdot \||$ on $Y$ is just the Minkowski functional of $C$. Then Lemma 4.3 says that there is $\varepsilon_{0}>0$ such that for any fixed $0<\varepsilon<\varepsilon_{0}$ there exists a norm $|\cdot|$ on $Y=\operatorname{span} C$ satisfying 
(a) $(1-\varepsilon)|||x||| \leq|x| \leq|\|x \mid\|$ for all $x \in Y$.

(b) For any two sequences $\left\{x_{n}\right\},\left\{y_{n}\right\}$ in $B_{Y}$ with $\left\|\mid x_{n}\right\| \| \rightarrow 1$ and $\left\|\left|y_{n}\right|\right\| \rightarrow 1,\left|x_{n}\right|+\left|y_{n}\right|-\left|x_{n}+y_{n}\right| \rightarrow 0$ implies $\left\|x_{n}-y_{n}\right\| \rightarrow 0$.

Now, applying Lemma 4.4, we claim that $|\cdot|$ is uniformly convex on $W$. To see this, let $\left\{x_{n}\right\},\left\{y_{n}\right\}$ be sequences in $B_{\||| \cdot||}(r) \cap W$ with $\left\|\mid x_{n}\right\| \| \rightarrow r$, $\left\|y_{n}\right\| \mid \rightarrow r$ and

$$
\left|x_{n}\right|+\left|y_{n}\right|-\left|x_{n}+y_{n}\right| \rightarrow 0 \text {. }
$$

By (b), we have $\left\|x_{n}-y_{n}\right\| \rightarrow 0$. Since the topologies generated by $\|\cdot\|$ and \|\|$\cdot \|$ coincide on $W$ by Lemma 4.7 , this says that $\left\|x_{n}-y_{n}\right\| \| \rightarrow 0$. This together with (a) and Lemma 4.4 implies that $|\cdot|$ is uniformly convex on $W$.

Next, in order to obtain our desired norm, we use the factorization theorem twice. Starting with the super-weakly compact set $C$ of $X$ and repeating the construction above, we again get a reflexive space $\left(E,\||\cdot|\|_{1}\right)$ with closed unit ball $C_{1} \supset C$ and a norm $|\cdot|_{1}$ equivalent to $\|\left.|\cdot|\right|_{1}$ on $E$ and uniformly convex on $C$. Finally, let $\|\cdot\|_{1}=\sqrt{|\cdot|^{2}+|\cdot|_{1}^{2}}$ on $E$. Then it is not difficult to check that $\|\cdot\|_{1}$ is equivalent to $|\cdot|$ on $E$ and has the desired properties.

Corollary 4.9. Suppose that $W$ is a closed bounded convex subset of a Banach space $X$. Then it is super-weakly compact if and only if there exists a uniformly continuous and uniformly convex function on $W$.

Proof. Sufficiency is obvious by Theorem 4.8.

Necessity. By Corollary 3.11, we can assume that $W$ is symmetric. Now, by Theorem 4.8 , there exists a norm $\|\cdot\|_{1}$ on $\operatorname{span} W$ which is uniformly continuous and uniformly convex on $W$. We complete the proof by taking $f=\|\cdot\|_{1}^{2}$.

From Theorem 4.8 we see that for every super-weakly compact convex symmetric set $W$ of a Banach space $(X,\|\cdot\|)$, there exists a reflexive Banach space $\left(E,\|\cdot\|_{1}\right)$ whose norm is stronger than $\|\cdot\|$ and $W$ is uniformly convex with respect to $\|\cdot\|_{1}$. In the following, we will start from $\|\cdot\|_{1}$ to construct an equivalent norm $|\cdot|$ on $X$ such that $W$ is uniformly convex with respect to $|\cdot|$, i.e. $W$ is uniformly convexifiable. We first need some preparations.

Let the Banach space $X$, the subset $W$, and the norms $\|\cdot\|$ and $\|\cdot\|_{1}$ be as in Theorem 4.8 with $\|\cdot\|_{1} \geq\|\cdot\|$ on $E$ and assume that $f$ is defined on $X$ by

$$
f(x)= \begin{cases}\|x\|_{1} & \text { if } x \in W \\ +\infty & \text { otherwise. }\end{cases}
$$

Clearly, $f$ is extended-real-valued lower semicontinuous and convex on $X$, and $f^{2}$ is uniformly convex on $W$.

For each integer $n \geq 1$ let $f_{n}$ be the inf-convolution of $f$ and $n\|\cdot\|$, i.e.

$$
f_{n}(x)=\inf \{f(y)+n\|x-y\|: y \in X\}, \quad x \in X .
$$


Lemma 4.10. The set $W$, the norm $\|\cdot\|$ and the sequence $\left\{f_{n}\right\}$ of infconvolutions as above satisfy:

(i) For every $n \geq 1,\|x\| \leq f_{n}(x) \leq n\|x\|$ for all $x \in X$.

(ii) $\left\{f_{n}\right\}$ is uniformly convergent to $f$ on $W$.

(iii) For every $\varepsilon>0, f_{n}^{2}$ is $\varepsilon$-uniformly convex for all sufficiently large $n \geq 1$.

Proof. (i) is trivial since $f \geq\|\cdot\|$ on $X$ and since $f_{n}$ is Lipschitzian with Lipschitz norm $L_{n} \leq n$ (see, for instance, [6, p. 24], or [21, 22]).

(ii) Suppose, to the contrary, that $a_{n}=\sup \left\{\left|f_{n}(x)-f(x)\right|: x \in W\right\}$ does not converge to zero. Then $\lim _{n \rightarrow \infty} a_{n}=a>0$ since $a_{n}=\sup \left\{f(x)-f_{n}(x)\right.$ : $x \in W\}$ is nonincreasing in $n$. Let $x_{n}, y_{n} \in W$ be such that

$$
f\left(x_{n}\right)-f_{n}\left(x_{n}\right) \geq 3 a / 4, \quad f_{n}\left(x_{n}\right) \geq f\left(y_{n}\right)+n\left\|x_{n}-y_{n}\right\|-a / 4 .
$$

Then for all $n \in \mathbb{N}$,

$$
f\left(x_{n}\right) \geq a / 2+f\left(y_{n}\right)+n\left\|x_{n}-y_{n}\right\| .
$$

Thus, $\left\|x_{n}-y_{n}\right\| \rightarrow 0$. Uniform continuity of $f$ on $W$ implies $f\left(x_{n}\right)-f\left(y_{n}\right) \rightarrow 0$. This contradicts $f\left(x_{n}\right)-f\left(y_{n}\right) \geq a / 2>0$.

It remains to show (iii). Note $f^{2}$ is a uniformly convex function on $W$. For every $\varepsilon>0$ there is $\delta>0$ satisfying

$$
f^{2}(x)+f^{2}(y)-2 f^{2}\left(\frac{x+y}{2}\right) \geq \delta
$$

whenever $\|x-y\| \geq \varepsilon$. Note that $f_{n}^{2}$ is uniformly convergent to $f^{2}$ on $W$. We can choose $K \in \mathbb{N}$ such that for all positive integers $n>K$ and for all $x \in W,\left|f_{n}^{2}(x)-f^{2}(x)\right|<\delta / 4$. We claim that $f_{n}^{2}$ is $\varepsilon$-uniformly convex on $W$ for all $n>K$. To see this, let $x, y \in W$ with $\|x-y\| \geq \varepsilon$. Then

$$
\begin{aligned}
f_{n}^{2}(x)+f_{n}^{2}(y)-2 f_{n}^{2}\left(\frac{x+y}{2}\right)=\left(f_{n}^{2}(x)-f^{2}(x)\right)+\left(f_{n}^{2}(y)-f^{2}(y)\right) & \\
-2\left(f_{n}^{2}\left(\frac{x+y}{2}\right)-f^{2}\left(\frac{x+y}{2}\right)\right) & +f^{2}(x)+f^{2}(y)-2 f^{2}\left(\frac{x+y}{2}\right) \\
& >\left(-\frac{\delta}{4}\right)+\left(-\frac{\delta}{4}\right)+0+\delta=\frac{\delta}{2} .
\end{aligned}
$$

This proves our assertion and completes the proof of the lemma.

Lemma 4.11. Let $W \subset(X,\|\cdot\|)$ be a super-weakly compact convex set. Then there exists a uniformly convex function $g$ on $W$ and positive numbers $\alpha$ and $\beta$ such that

$$
\alpha\|x\|^{2} \leq g(x) \leq \beta\|x\|^{2} \quad \text { for all } x \in X .
$$


Proof. We can assume that $W$ is symmetric. For any fixed $\varepsilon>0$, we let $\varepsilon_{k}=\varepsilon / 2^{k}$ and let $\left\{f_{n}\right\}$ be the sequence of inf-convolutions defined above. For every $k$, applying Lemma 4.10 , we can choose $n_{k} \in \mathbb{N}$ such that $f_{n_{k}}^{2}$ is $\varepsilon_{k}$-uniformly convex on $W$. Now, let

$$
g(x)=\sum_{k=1}^{\infty} 2^{-k} n_{k}^{-2} f_{n_{k}}^{2}(x) \quad \text { for all } x \in X
$$

Then $g$ is as claimed.

We can now prove the main result of this paper.

TheOREM 4.12. Let $W \subset(X,\|\cdot\|)$ be a bounded closed convex set. Then $W$ is super-weakly compact if and only if $W$ is uniformly convexifiable.

Proof. The "if" part follows immediately from Theorem 3.8 or Corollary 4.9 .

The "only if" part: We assume again that $W$ is symmetric. Let $g$ be a function on $W$, and $\alpha$ and $\beta$ be positive numbers such that

$$
\alpha\|x\|^{2} \leq g(x) \leq \beta\|x\|^{2} \quad \text { for all } x \in X .
$$

For $m \geq 1$ and $x \in X$, define $h_{m}(x)=m^{2} g(x / m)$. Then it is easy to see that for all $m \geq 1, h_{m}$ is uniformly convex on $m W$, and $\alpha\|x\|^{2} \leq h_{m}(x) \leq \beta\|x\|^{2}$ for all $m \geq 1$ and all $x \in X$. Take

$$
h(x)=\sum_{m=1}^{\infty} \frac{1}{2^{m}} h_{m}(x) \quad \text { for all } x \in X .
$$

Obviously, $h$ is uniformly convex on each fixed $m W(m \in \mathbb{N})$ and

$$
\alpha\|x\|^{2} \leq h(x) \leq \beta\|x\|^{2} \quad \text { for all } x \in X .
$$

Put $B=\{x \in X: h(x) \leq 1\}$. Finally, let $p$ be the Minkowski functional of $B$. Then $p$ is an equivalent norm on $X$, and we claim that the norm $p$ is uniformly convex on $W$. To see this, let $x_{n}, y_{n}$ in $W$ be such that

$$
p\left(x_{n}\right) \rightarrow r, \quad p\left(y_{n}\right) \rightarrow r, \quad p\left(\frac{x_{n}+y_{n}}{2}\right) \rightarrow r .
$$

Next, let $x_{n}^{\prime}=x_{n} / r$ and $y_{n}^{\prime}=y_{n} / r$. Then $x_{n}^{\prime}, y_{n}^{\prime} \in m W$ for all sufficiently large $m \in \mathbb{N}$ with $m \geq 1 / r$. Thus $p\left(x_{n}^{\prime}\right) \rightarrow 1, p\left(y_{n}^{\prime}\right) \rightarrow 1$, and $p\left(\left(x_{n}^{\prime}+y_{n}^{\prime}\right) / 2\right) \rightarrow 1$. Therefore

$$
h\left(x_{n}^{\prime}\right) \rightarrow 1, \quad h\left(y_{n}^{\prime}\right) \rightarrow 1, \quad h\left(\frac{x_{n}^{\prime}+y_{n}^{\prime}}{2}\right) \rightarrow 1 .
$$

The uniform convexity of $h$ on $m W$ implies $\left\|x_{n}^{\prime}-y_{n}^{\prime}\right\| \rightarrow 0$. The equivalence of the norms $p$ and $\|\cdot\|$ in turn implies $p\left(x_{n}^{\prime}-y_{n}^{\prime}\right) \rightarrow 0$, and further $p\left(x_{n}-y_{n}\right)$ $\rightarrow 0$. Therefore $p$ is the desired norm. 
Acknowledgements. We want to thank the referee for detailed comments which led to a number of improvements. We are also grateful to the Editor, Professor Tadeusz Figiel, for his helpful suggestions and corrections. We thank Professor Xin Zhouping for his support when the first named author was visiting in the Institute of Mathematical Sciences of the Chinese University of Hong Kong. We also want to thank Professor Jiang Chunlan and Professor Chen Xiaoman for helpful conversations about this paper.

This research was supported by NSFC grant no. 10771175 and by the Institute of Mathematical Sciences at the Chinese University of Hong Kong.

\section{References}

[1] B. Beauzamy, Introduction to Banach Spaces and Their Geometry, 2nd ed., NorthHolland Math. Stud. 68, North-Holland, Amsterdam, 1985.

[2] Y. Benyamini and J. Lindenstrauss, Geometric Nonlinear Functional Analysis, Vol. 1, Amer. Math. Soc. Colloq. Publ. 48, Amer. Math. Soc., Providence, RI, 2000 .

[3] L. Cheng, C. Wu, X. Xue and X. Yao, Convex functions, subdifferentiability and renormings, Acta Math. Sinica (N.S.) 14 (1998), 47-56.

[4] J. A. Clarkson, Uniformly convex spaces, Trans. Amer. Math. Soc. 40 (1936), 396414.

[5] W. J. Davis, T. Figiel, W. B. Johnson and A. Pełczyński, Factoring weakly compact operators, J. Funct. Anal. 17 (1974), 311-327.

[6] R. Deville, G. Godefroy and V. Zizler, Smoothness and Renormings in Banach Spaces, Pitman Monogr. Surveys Pure Appl. Math. 64, Longman Sci. \& Tech. and Wiley, New York, 1993.

[7] J. Diestel, Geometry of Banach Spaces-Selected Topics, Lecture Notes in Math. 485, Springer, Berlin, 1975.

[8] J. Diestel and J. J. Uhl, Vector Measures, Math. Surveys 15, Amer. Math. Soc., Providence, RI, 1977.

[9] P. Enflo, Banach spaces which can be given an equivalent uniformly convex norm, Israel J. Math. 13 (1972), 281-288.

[10] G. Godefroy, Renormings of Banach spaces, in: Handbook of the Geometry of Banach Spaces, Vol. I, North-Holland, Amsterdam, 2001, 781-835.

[11] K. Goebel and W. A. Kirk, Topics in Metric Fixed Point Theory, Cambridge Stud. Adv. Math. 28, Cambridge Univ. Press, Cambridge, 1990.

[12] R. C. James, Weak compactness and reflexivity, Israel J. Math. 2 (1964), 101-119.

[13] -, Super-reflexive Banach spaces, Canad. J. Math. 24 (1972), 896-904.

[14] - Some self-dual properties of normed linear spaces, in: Proc. Sympos. Infinite Dimensional Topology, Ann. of Math. Stud. 69, Princeton Univ. Press, 1972, 159175 .

[15] R. C. James and J. J. Schäffer, Super-reflexivity and the girth of spheres, Israel J. Math. 11 (1972), 398-404.

[16] W. B. Johnson and J. Lindenstrauss (eds.), Handbook of the Geometry of Banach Spaces, Vol. I, North-Holland, Amsterdam, 2001.

[17] G. Kasparov and G. Yu, The coarse geometric Novikov conjecture and uniform convexity, Adv. Math. 206 (2006), 1-56. 
[18] W. A. Kirk, A fixed point theorem for mappings which do not increase distances, Amer. Math. Monthly 72 (1965), 1004-1006.

[19] J. Lindenstrauss and L. Tzafriri, Classical Banach Spaces II, Ergeb. Math. Grenzgeb. 97, Springer, Berlin, 1979.

[20] G. Pisier, Martingales with values in uniformly convex spaces, Israel J. Math. 20 (1975), 326-350.

[21] C. Wu and L. Cheng, On extensions of the Preiss differentiability theorem, J. Funct. Anal. 124 (1994), 184-193.

[22] - , - A note on the differentiability of convex functions, Proc. Amer. Math. Soc. 121 (1994), 1057-1062.

Lixin Cheng, Qingjin Cheng (corresponding author), Bo Wang, Wen Zhang

Department of Mathematics

Xiamen University

Xiamen 361005, China

E-mail: lxcheng@xmu.edu.cn

qjcheng@xmu.edu.cn

mikle4505@yahoo.com.cn

wenzhang@xmu.edu.cn

Received July 7, 2009

Revised version May 9, 2010 